\title{
Transcutaneous application of ultrasound enhances the effects of finasteride in a murine model of androgenic alopecia
}

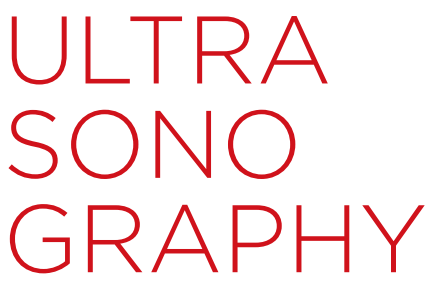

\author{
Jaeho Kim ${ }^{1,2}$, Hyun-Chul Kim ${ }^{1}$, Kavin Kowsari ${ }^{1}$, Kyungho Yoon ${ }^{3}$, Seung-Schik Yoo ${ }^{1}$ \\ 'Department of Radiology, Brigham and Women's Hospital, Harvard Medical School, Boston, \\ MA, USA; ${ }^{2}$ Department of Neurology, Dongtan Sacred Heart Hospital, Hallym University \\ College of Medicine, Hwaseong; ${ }^{3}$ Center for Healthcare Robotics, Korea Institute of Science \\ and Technology, Seoul, Korea
}

Purpose: The purpose of this study was to evaluate if transcutaneous application of low-intensity ultrasound can locally enhance the effects of finasteride on hair growth in a murine model of androgenic alopecia (AA).

Methods: AA mice (injected twice per week with testosterone enanthate, $n=11$ ), under daily oral administration of finasteride, received 1-MHz ultrasound for 1 hour at the unilateral thigh area five times per week for 5 weeks. Non-thermal and non-cavitational ultrasound was delivered in a pulsed manner (55-ms pulse duration with a repetition frequency of $4 \mathrm{~Hz}$ ). Skin temperature was measured during sonication, and the measurements were validated with numerical simulations of sonication-induced tissue temperature changes. Hair growth was assessed both photographically and histologically.

Results: We found more hair growth on the sonicated thigh area than on the unsonicated thigh, beginning from week 3 through the end of the experiment. Histological analyses showed that the number of hair follicles doubled in the skin sections that received sonication compared to the unsonicated zone, with thicker follicular diameter and skin. An over five-fold increase was also observed in the anagen/telogen ratio in the sonicated area, suggesting an enhanced anagen phase. Skin temperature was unaltered by the administered sonication.

Conclusion: The findings of the present study suggest that pulsed application of ultrasound promotes hair growth, potentially by disrupting the binding of albumin to finasteride. This may suggest further applications to enhance the pharmacological effects of other relevant drugs exhibiting high plasma protein binding.

Keywords: Ultrasound; Finasteride; Albumin; Androgenic alopecia; Plasma protein binding Key points: Pulsed application of ultrasound reversibly unbinds pharmacological agents from plasma proteins. Transcutaneous application of low-intensity ultrasound locally enhances the effects of finasteride on hair growth from a murine model of androgen alopecia. The given ultrasound is non-cavitational and non-thermal.

Received: September 5, 2021

Revised: November 5, 2021

Accepted: November 10, 2021

Correspondence to:

Seung-Schik Yoo, PhD, MBA, Department of Radiology, Brigham and Women's Hospital, Harvard Medical School, 75 Francis Street, Boston, MA 02115, USA

Tel. +1-617-732-9464

Fax. +1-617-732-9082

E-mail: yoo@bwh.harvard.edu

This is an Open Access article distributed under the terms of the Creative Commons Attribution NonCommercial License (http://creativecommons.org/ licenses/by-nc/4.0/) which permits unrestricted noncommercial use distribution, and reproduction in any medium, provided the original work is properly cited.

Copyright @ 2022 Korean Society of Ultrasound in Medicine (KSUM)

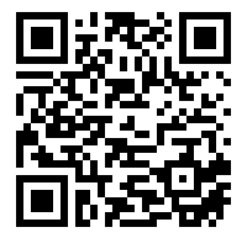

How to cite this article:

Kim J, Kim HC, Kowsari K, Yoon K, Yoo SS Transcutaneous application of ultrasound enhances the effects of finasteride in a murine model of androgenic alopecia. Ultrasonography. 2022 Apr;41(2):382-393. 


\section{Introduction}

Androgenic alopecia (AA), also known as 'male-pattern baldness', stems from the reaction of hair follicles to circulating testosterone and its derivatives, resulting in progressive hair loss. It is the most common cause of hair loss in both men and women [1]. Nonpharmacological therapeutic options for AA include scalp microneedling [2], electrical stimulation of the scalp [3], and photic scalp stimulation [4]. However, these non-pharmacological treatments have a limited effect on AA. Minoxidil, a popular topical pharmacological agent, is known to increase the amount of intracellular calcium ions [5], which have been shown to upregulate adenosine triphosphate (ATP) synthase activity, thereby promoting hair growth [6]. Although it has fewer side effects than finasteride [7], minoxidil must be used long-term and is significantly less effective than oral finasteride treatment [8]. Finasteride inhibits the action of 5a-reductase-in particular, 5a-reductase type II, which is highly expressed in the hair follicles in the scalp of AA individuals [9]. 5a-reductase plays an important role in converting testosterone to dihydrotestosterone (DHT), which accelerates hair loss (the mechanism of DHT in hair loss is discussed in detail elsewhere [10]). Finasteride has several side effects, including decreased libido and erectile/ejaculatory dysfunction [11], all of which continue after cessation of the drug [12]. Finasteride also artificially lowers blood prostate-specific antigen levels [13], which may decrease the sensitivity of prostate cancer detection. In some cases, finasteride may also lead to kidney damage [14]. To reduce these pharmacological side effects, efficient delivery of finasteride to the scalp while minimizing systemic exposure is warranted.

Recent investigations have revealed that pulsed application of acoustic pressure waves can unbind pharmacological agents from plasma proteins $[15,16]$. For example, an anti-epileptic drug, phenytoin, was unbound from albumin by the focal application of ultrasound, enhancing its regional parenchymal uptake across the blood-brain barrier in rodents [15]. More recently, ultrasound was also used to unbind lidocaine from a1-acid glycoproteins to enhance local anesthetic effects in rats [16]. The radiation force conferred by the application of acoustic pressure waves to biological tissue, below the threshold for induced thermal and cavitational effects, is believed to disrupt the weak binding forces between plasma proteins and the drug, thus temporarily enhancing the bioavailability of unbound ('free') drugs for their action [16].

The utility of the ultrasound technique in unbinding pharmacological agents, such as a type of drug that modulates enzymatic action, has not been demonstrated. Finasteride readily binds to albumin in blood, with a $>90 \%$ binding rate [17], and the resulting albuminfinasteride complex is not easily transported across the capillary, limiting its pharmacological action. Thus, the authors hypothesized that non-invasive application of pulsed ultrasound could unbind finasteride from plasma protein, allowing higher levels of finasteride to be delivered to the skin, which would promote hair growth. The present study examined the effects of the application of ultrasound using a murine model of AA under a finasteride regimen. As ultrasound may induce tissue cavitation or temperature elevation (which may increase local blood flow), both of which could confound the interpretation of the results [18] or have ramifications involving undesired biological effects such as skin injury, premature skin aging, and neuropathic pain $[19,20]$, this study used noncavitational, low-intensity ultrasound that does not elevate the temperature of the sonicated tissue. The degree of hair growth was evaluated using photographic and histological analyses (hematoxylin and eosin $[\mathrm{H \& E}]$ staining and $\beta$-catenin staining).

\section{Materials and Methods}

\section{Compliance with Ethical Standards}

Experiments were conducted under the approval of and according to the regulations and standards set forth by the Institutional Animal Care and Use Committee (IACUC).

\section{Animal Preparation and Study Overview}

Six-week-old C57BL/6 mice ( $n=11$; all male, Charles River Laboratories, Wilmington, MA, USA), following a week of acclimation, entered the experimental procedures at 7 weeks of age for steady-state hair growth [21]. The average body weight at the onset of the experimental procedure was $21.9 \pm 1.6 \mathrm{~g}(\mathrm{n}=11)$.

An overview of the study's procedure is illustrated in Fig. 1. First, after removing fur on the back, mice received intramuscular injections of testosterone enanthate for 5 weeks to create the AA model. During this time, finasteride was orally given to the animals daily while ultrasound was administered to a unilateral thigh area, five times per week. Photographs were taken every week to track the degree of hair growth. Upon completion of the 5-week study period, animals were sacrificed, and the skin was harvested for histological analysis. The experimental procedures are described in detail below.

\section{Androgenic Alopecia Model}

Under anesthesia of ketamine and xylazine (ketamine:xylazine $=100: 10 \mathrm{mg} / \mathrm{kg}$, intraperitoneal injection), the fur on the animal's back was removed from the scapular line to the root of the tail, including the thighs of the hind limbs, using an electric clipper, followed by a 2-minute application of $\sim 2 \mathrm{~mL}$ of depilation lotion (Nair, Church \& Dwight Co., Inc., Ewing, NJ, USA) on the skin. The 


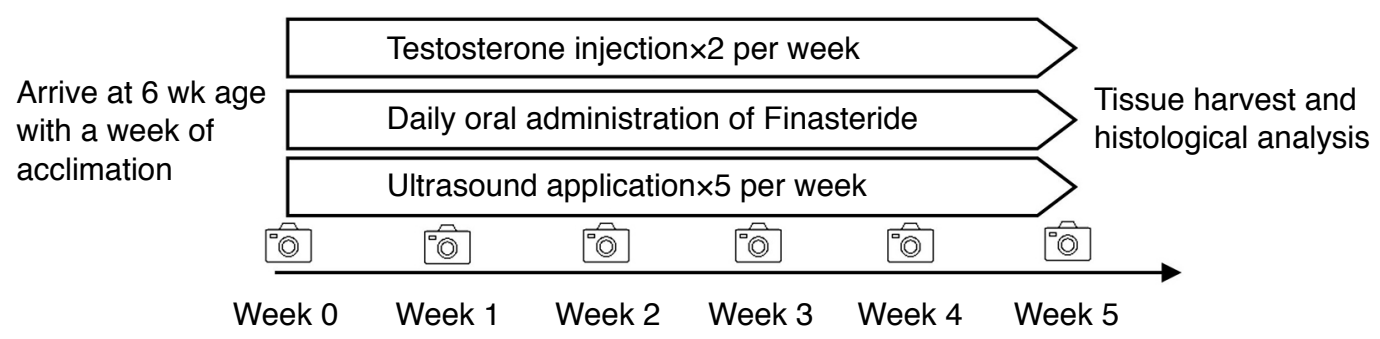

Fig. 1. Schematic overview of the experimental procedure. After a week of acclimation and subsequent hair removal, testosterone enanthate was intramuscularly injected into mice twice per week for 5 weeks while finasteride was orally given daily. Ultrasound was administered to a unilateral thigh area five times per week and photographs were taken weekly to track the degree of hair growth.

applied lotion was thoroughly removed with saline swabs. Then, testosterone enanthate (1648004, Sigma, St. Louis, MO, USA, suspended in sesame oil) was intramuscularly injected into the thigh muscle at a dose of $50 \mathrm{mg} / \mathrm{kg}$, twice weekly (with a 3-day gap between injections) for 5 weeks. Testosterone enanthate was chosen due to its longer pharmacological half-life (i.e., 4-5 days) than other testosterone-based agents that require more frequent injections [22]. The side of the injection was randomized and balanced.

\section{Administration of Finasteride}

Finasteride (Propecia, Merck, Kenilworth, NJ, USA) was suspended in distilled water and orally administered at $0.5 \mathrm{mg} / \mathrm{kg}$ via a gavage tube (FTP-20-38-50, Instech Laboratory, Plymouth Meeting, PA, USA), once daily throughout the entire experimental duration (i.e., 5 weeks). Before the application of each dose, an aqueous finasteride suspension was mixed to maintain homogeneous dose levels at room temperature.

\section{Ultrasound Application}

The animals were anesthetized under isoflurane ( $2 \%$ induction, $\sim 1 \%$ for maintenance) and ultrasound was administered to the right thigh area unilaterally (Fig. 2A, B). Ultrasound gel (Aquasonic, Parker Laboratory, Fairfield, NJ, USA) was applied to the skin to provide acoustic coupling. An ultrasound transducer (V302-SU, Olympus America Inc., Waltham, MA, USA) operating at a fundamental frequency of $1 \mathrm{MHz}$ was used to deliver ultrasound in a pulsed manner ( $55 \mathrm{~ms}$ pulse duration, $4 \mathrm{~Hz}$ pulse repetition frequency, at a $2.5 \mathrm{~W} / \mathrm{cm}^{2}$ spatial peak pulse average acoustic intensity, $\left.1_{\text {SPPA }}\right)$. The ultrasound transducer, which had a $28.4 \mathrm{~mm}$ outer diameter, was actuated by a sinusoidal electrical signal (33210A, Agilent Technologies, Santa Clara, CA, USA) amplified by a linear power amplifier (240L, Electronics and Innovations Ltd., Rochester, NY, USA) with impedance-matching (T-200, Electronics and Innovations Ltd.). The acoustic intensity from the transducer surface with respect to the magnitude of input voltage was calibrated using a calibrated hydrophone (HNR500, Onda, Sunnyvale, CA, USA) $5 \mathrm{~mm}$ away from the transducer surface (estimated distance to the skin). The acoustic intensity profile, measured $1 \mathrm{~mm}$ away from the transducer surface, was uniform across the field (Fig. 2C). The corresponding peak-topeak pressure amplitude was $549 \mathrm{kPa}$. A duty cycle of $22 \%$ yielded a spatial peak temporal average acoustic intensity $\left(I_{\text {SPTA }}\right)$ of $550 \mathrm{~mW} /$ $\mathrm{cm}^{2}$. The choice of the acoustic pulsing parameter was based on previous studies that showed unbinding of phenytoin and lidocaine from plasma proteins $[15,16]$. The mechanical index, rarefaction pressure $\left(\mathrm{P}_{\mathrm{r}} \text { in } \mathrm{MPa} \text { ) } \times \text { fundamental frequency (in } \mathrm{MHz}\right)^{-0.5}$, indicates the risk of mechanical damage to the tissue, and a value of 1.9 is currently defined as the regulatory limit for most ultrasound imagers [23]. In the present study, the mechanical index of the ultrasound was $0.225 \mathrm{MPa} \times 1 \mathrm{MHz}^{-0.5}=0.225$. Sonication was given for 1 hour, five times per week for 5 weeks (a total of 25 sonication sessions).

\section{Assessment of Hair Growth and Image Analysis}

Hair growth was photographed weekly, starting on the day of skin preparation, for 5 weeks with the same field-of-view and lighting conditions (Galaxy Note 8, Samsung Electronics, Seoul, Korea), with a unified pixel dimension (200 $\mu \mathrm{m} /$ pixel) across the animals. To qualitatively illustrate the group-averaged hair growth pattern in the presence of unavoidable variability in animals' postures at the time of capturing photographs, the images were aligned to one another, being spatially referenced to the root of the tail along the midline. The aligned images from 11 mice were then overlaid with an adjustment of transparency (to $91 \%$ ) to generate a single composite image representing the group-averaged hair growth pattern at each time point. The resulting composite images were converted to an 8-bit grayscale image (pixel intensity range of $0-255)$ and thresholded at an intensity value of 128 to accentuate the area of hair growth. This image processing method, which was inherently sensitive to the threshold level selection, was intended for a qualitative group-level visualization of the area of hair growth and required animal-specific region-of-interest (ROI) evaluation.

For the ROI analysis, the background pixels outside of the animal were cropped and the mean pixel intensity value was calculated 


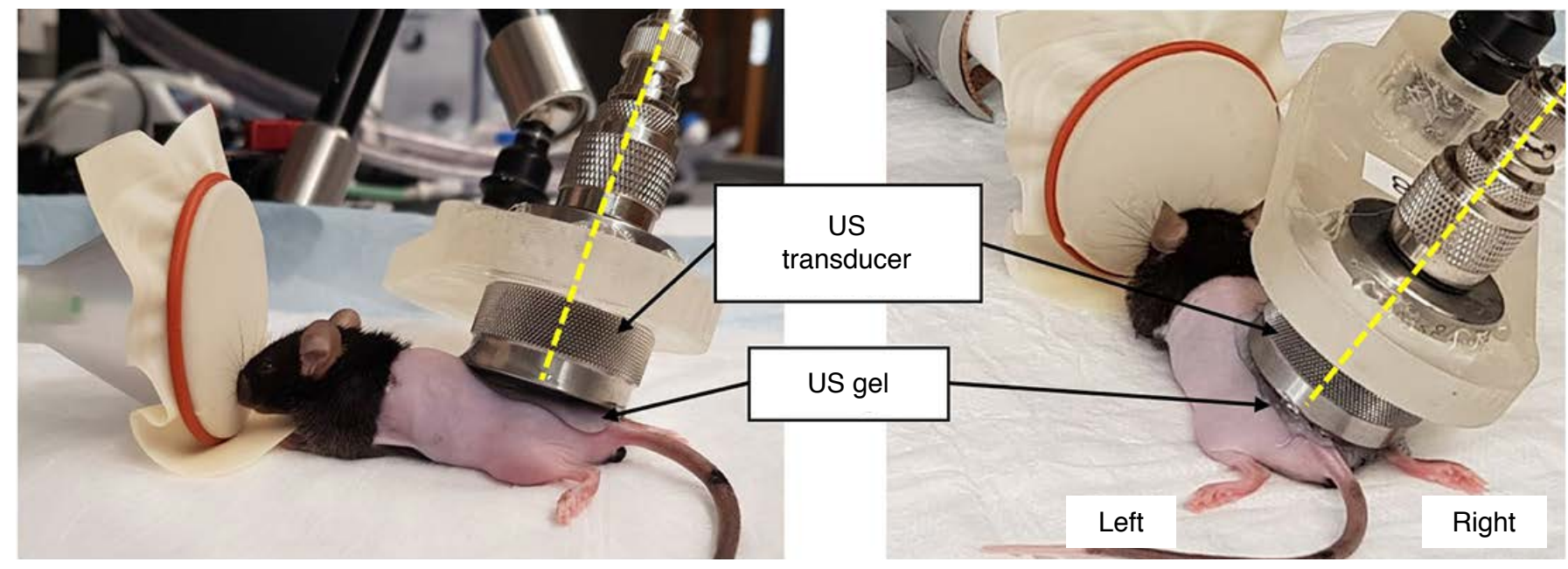

A
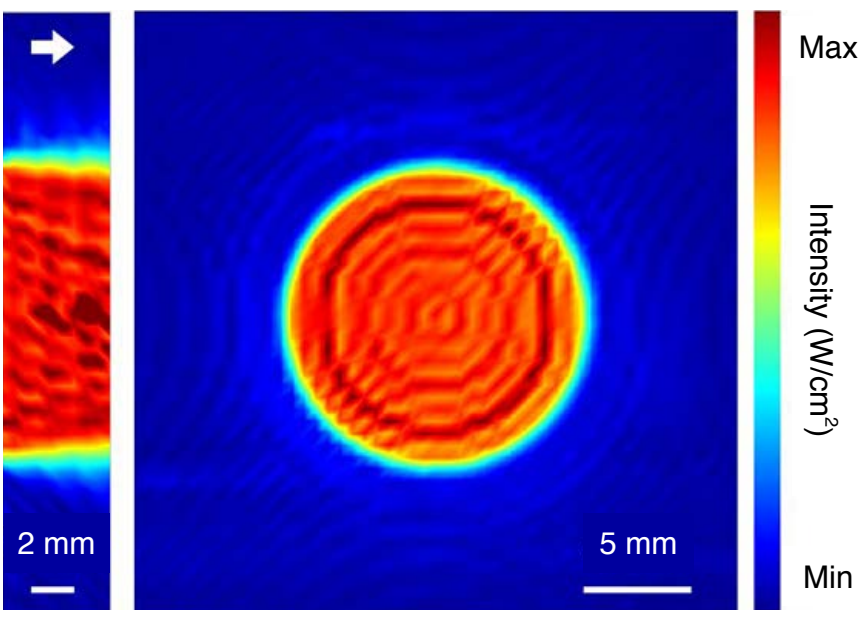

C

from a circular region (diameter $=40$ pixels) at the unshaved back between the scapulae. Then, an intensity threshold (10 times the mean pixel intensity from the bare shaved skin) was applied to delineate the area of hair growth. The ratio of hair growth and bare skin was then calculated from two rectangular ROls symmetrically placed to include the sonicated thigh area (height $\times$ width $=100 \times 40$ pixels, corresponding to an area of $\left.20 \times 0.8 \mathrm{~mm}^{2}\right)$. Although the sonication was applied in a circular area of the skin, a rectangular ROI was chosen to include the skin area used for subsequent histological sectioning and analysis.

\section{Histological Assessment of Hair Growth}

Upon completion of the experiment, animals were sacrificed using a fatal dose of intra-peritoneal injection of pentobarbital $(100 \mathrm{mg} / \mathrm{kg})$ under anesthesia of ketamine and xylazine (ketamine: $x y l a z i n e=100: 10 \mathrm{mg} / \mathrm{kg}$ ). Then, the skin on the back, encompassing the shoulders and thighs, was removed and immersed
Fig. 2. Sonication setup and acoustic intensity profile.

A. Sonication setup viewed from the side of an androgenic alopecia mouse is shown with application of ultrasound (US) gel for acoustic coupling between the transducer and the skin. The yellow dashed line represents the axial path of sonication originating from the transducer. B. The same setup viewed from the rear of the mouse. C. The acoustic intensity profile was measured $1 \mathrm{~mm}$ away from the exit plane of the transducer on the longitudinal (left; $30 \mathrm{~mm} \times 10$ $\mathrm{mm}$; 1 -mm step size) and transverse (right; $30 \mathrm{~mm} \times 30 \mathrm{~mm}$; 1-mm step size) planes (right). The arrow indicates the direction of sonication.

in $10 \%$ formaldehyde for 24 hours and then in $70 \%$ ethyl alcohol for 24 hours. The rectangular skin areas (dimensions; $3 \mathrm{~mm} \times 6 \mathrm{~mm}$ ) within the rectangular $\mathrm{ROI}$ for image analysis were removed from the skin and sliced into 6 - $\mu$ m-thick sections: one transverse and the other longitudinal with respect to the hair follicle orientation, as illustrated in Fig. 3. The skin sections were subjected to H\&E and $\beta$ -catenin staining. The expression of $\beta$-catenin indicates an enhanced level of the anagen phase of hair follicles [24]. For $\beta$-catenin staining, anti- $\beta$-catenin (sc-1496, Santa Cruz Biotechnology, Inc., Dallas, TX, USA) primary antibody was used for incubation overnight at $4^{\circ} \mathrm{C}$. After washing the slide, a biotin-conjugated secondary antibody (B9655, Sigma) and avidin (A9275, Sigma) were incubated to form an avidin-biotin complex and labeled with chromogen. The number and diameter of hair follicles, skin thickness, and the follicular anagen/telogen (A/T) ratio from both sonicated and unsonicated skin were quantified using the paired t-test (one-tailed). All analyses were performed using SPSS version 21 (IBM Corp., 


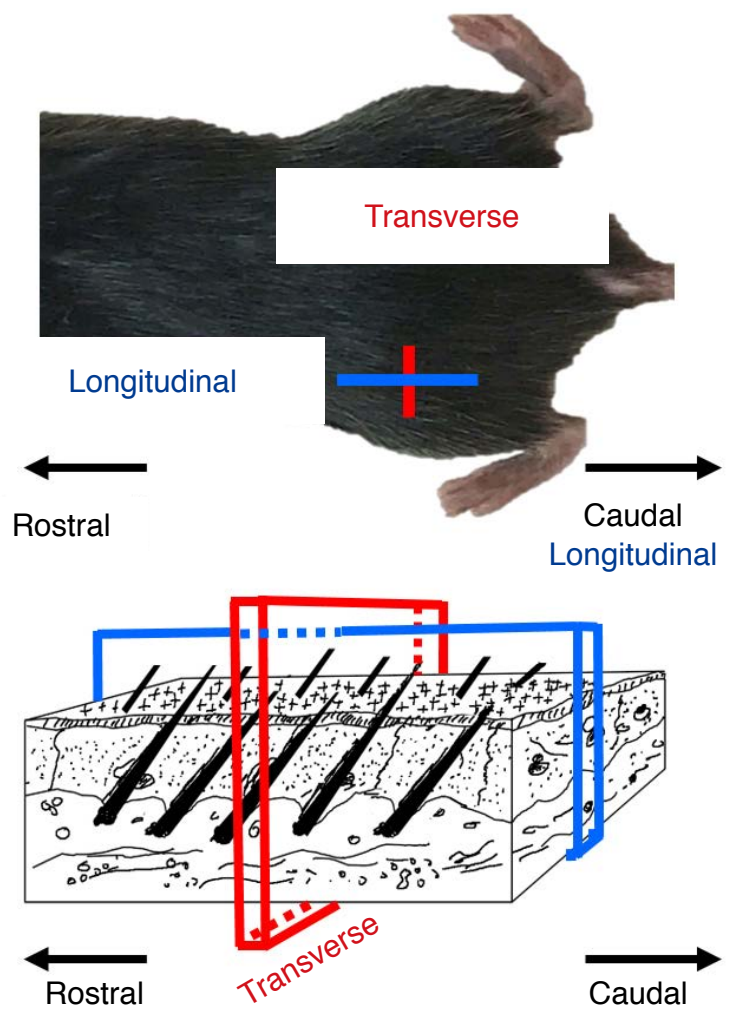

Fig. 3. Illustration of skin histological sectioning. The spatial orientation for histological sectioning along the transverse and longitudinal directions is illustrated (not to scale) from the rectangular region-of-interest.

Armonk, NY, USA).

\section{Assessment of Temperature Changes at the Skin and via} Thermal Simulation

Given that skin temperature may have an impact on local blood perfusion and concurrent hair growth [25], the local temperature at the skin surface was measured using an infrared thermal camera with a sensing sensitivity of $\sim 0.5^{\circ} \mathrm{C}$ (C3, FLIR Systems Inc., Wilsonville, OR, USA) every 15 minutes during the sonication procedure. In addition, numerical simulations of potential thermal effects from the sonication were conducted by sequentially solving the Khokhlov-Zabolotskaya-Kuznetsov and bio-heat transfer equations [26] through an open-source high-intensity ultrasound simulator based on MATLAB scripts (https://www.fda.gov/aboutfda/cdrh-offices/hitu-simulator) [27]. The estimation was performed using the maximum in situ acoustic intensity of $2.5 \mathrm{~W} / \mathrm{cm}^{2} I_{\text {SPPA }}$ for both the skin and underlying muscle, with a temporal resolution of $0.2 \mathrm{~ms}$. The thermal properties of the skin (specific heat of 3,300 $\mathrm{J} / \mathrm{kg} / \mathrm{K}$, thermal conductivity of $0.45 \mathrm{~W} / \mathrm{m} / \mathrm{K}$, and perfusion rate of $0.0013 \mathrm{~kg} / \mathrm{m}^{3} / \mathrm{s}$ ) and the muscle (specific heat of $3,465 \mathrm{~J} / \mathrm{kg} / \mathrm{K}$, thermal conductivity of $0.5 \mathrm{~W} / \mathrm{m} / \mathrm{K}$, perfusion rate of $0.009 \mathrm{~kg} / \mathrm{m}^{3} / \mathrm{s}$ )
[28] were used with the initial body temperature set at $36.5^{\circ} \mathrm{C}$.

\section{Results}

\section{Application of Ultrasound Promoted Hair Growth from AA Mice under Finasteride}

Weekly photographs in color, 8-bit grayscale, and thresholded binary composite images taken from the mice are shown in Fig. 4A-C respectively. Although there was animal-specific variability in terms of the location and degree of hair growth, the hair was qualitatively visible on the back and dorsal thigh areas starting from week 2. At week 5, the thigh area exposed to ultrasound (right, in green) grew more hair in the sonicated area (right thigh) than the unsonicated side. The thresholded binary images (Fig. 4C) showed that hair growth from the sonicated (right) thigh area contributed to more than half of the total pixel intensity in the composite image, whereas hair growth on the opposite thigh area did not.

Based on an animal-specific image-based ROI analysis, from week 3 to the end of the experiment, there was increased hair growth (in terms of the percentage of area of hair growth in the ROI) at the sonicated right thigh area compared to the unsonicated left thigh (paired t-test, $d f=10: P=0.024$ [week 3], $P=0.001$ [week 4], $P<0.001$ [week 5]) (Fig. 4D). At week 5, on average, regrown hair occupied $70.1 \% \pm 28.0 \%$ of the sonicated area, in contrast to only $50.4 \% \pm 36.9 \%$ in the unsonicated thigh area. No abnormalities were found in the sonicated/control skin over the study period.

\section{Histological Assessment of Hair Growth}

The hair follicles and skin thickness in the transverse and longitudinal sections (illustrated in Fig. 3) were evaluated after 5 weeks (exemplar H\&E-stained sections shown in Fig. 5). The number and diameter of the hair follicles were greater within the sonication area than in the area without any sonication. The area under the ultrasound exposure appeared to be thicker than its counterpart. The expression of $\beta$-catenin, an indicator of the anagen phase of hair follicles, was more prominent in the sonicated area than in the unsonicated region.

A quantitative analysis showed that the number (mean \pm standard deviation, $32.4 \pm 17.5)$ and diameter $(64.8 \pm 12.4 \mu \mathrm{m})$ of hair follicles from skin sections that received sonication were greater than those obtained from unsonicated areas $(16.1 \pm 10.0 \mu \mathrm{m}$ and $49.1 \pm 2.9$ $\mu \mathrm{m}$, respectively, for number and diameter of hair follicles; paired t-test, $d f=10, P=0.009$, Fig. $6 A ; d f=3, P=0.017$, Fig. 6B). The skin was thicker in the sonicated area $(329.4 \pm 51.2 \mu \mathrm{m}$ vs. $279.9 \pm 53.6$ $\mu \mathrm{m}$; paired $\mathrm{t}$ test, $\mathrm{df}=10, \mathrm{P}=0.021$ ) (Fig. $6 \mathrm{C}$ ) with a higher follicular $A / T$ ratio ( $0.9 \pm 0.9$ vs. $0.2 \pm 0.3$; paired t test, $d f=10, P=0.007$ ) (Fig. $6 D)$, suggesting an increased number of anagen hair follicles in the 

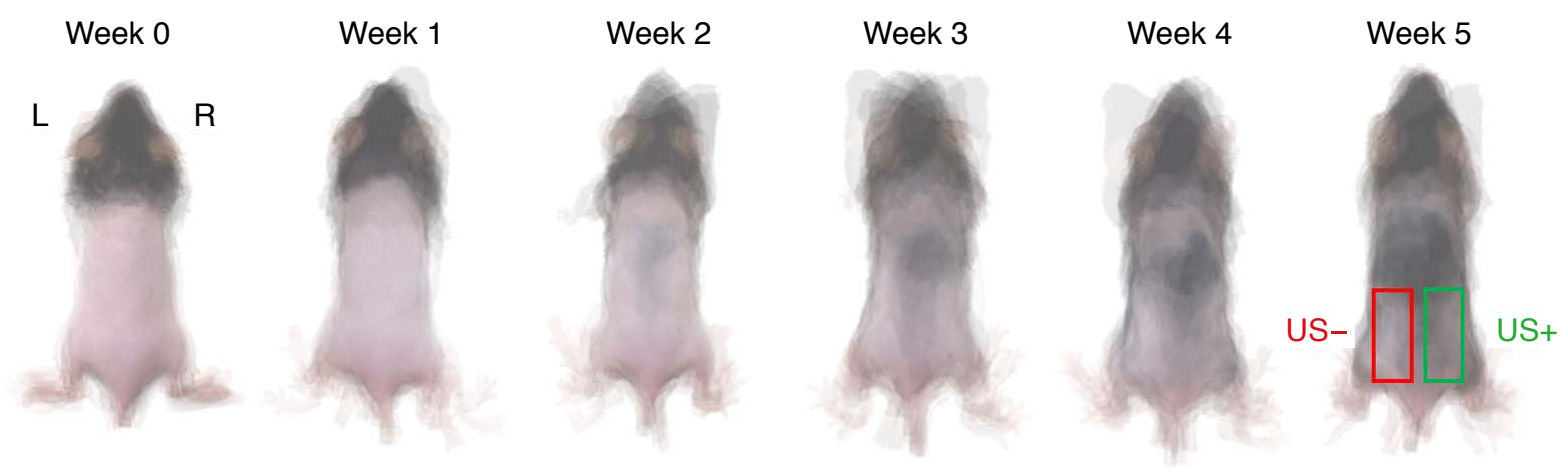

A
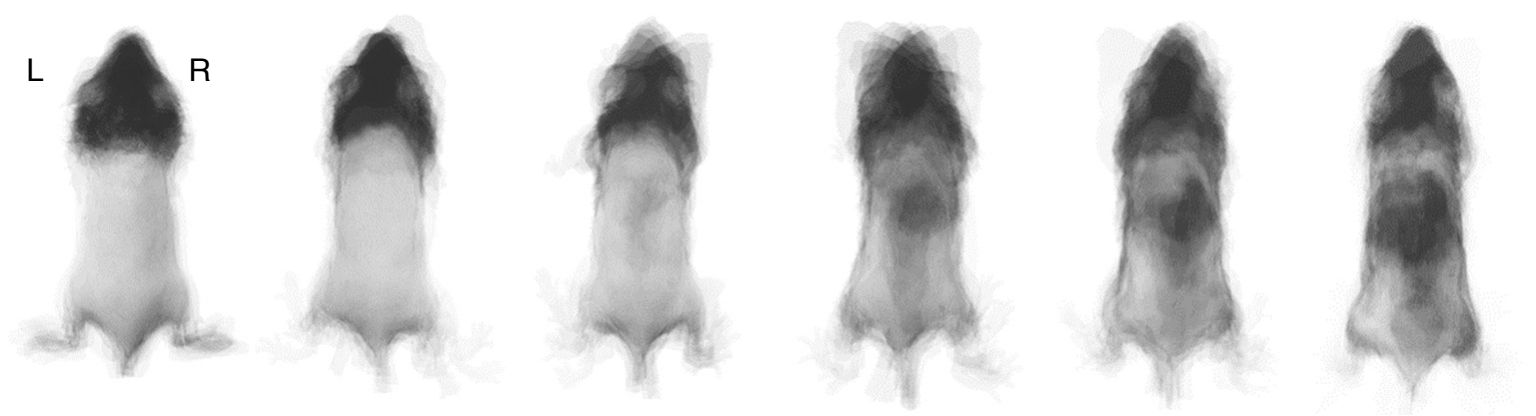

B

$\mathrm{L}$
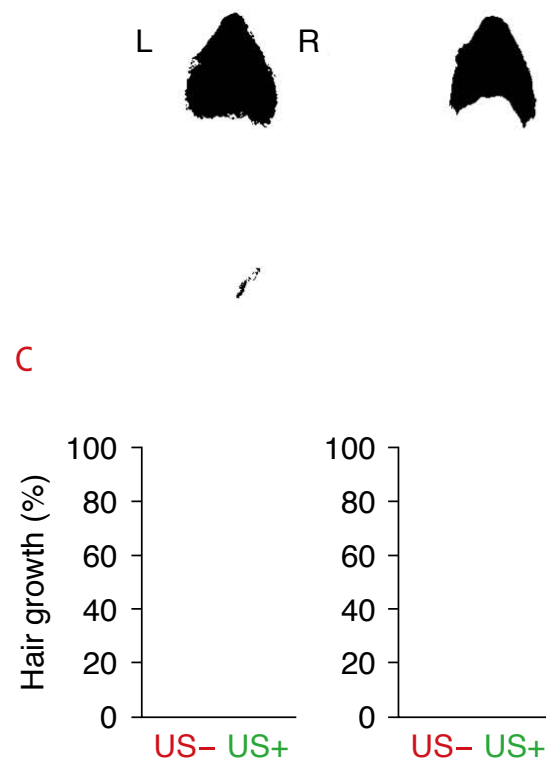

D
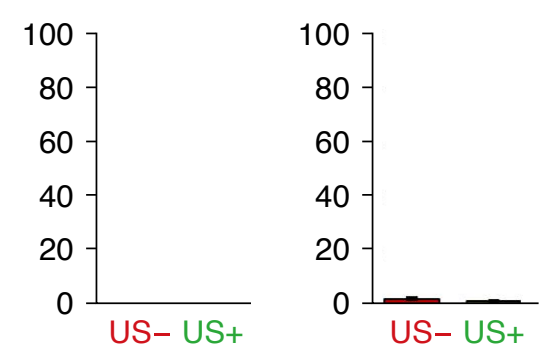
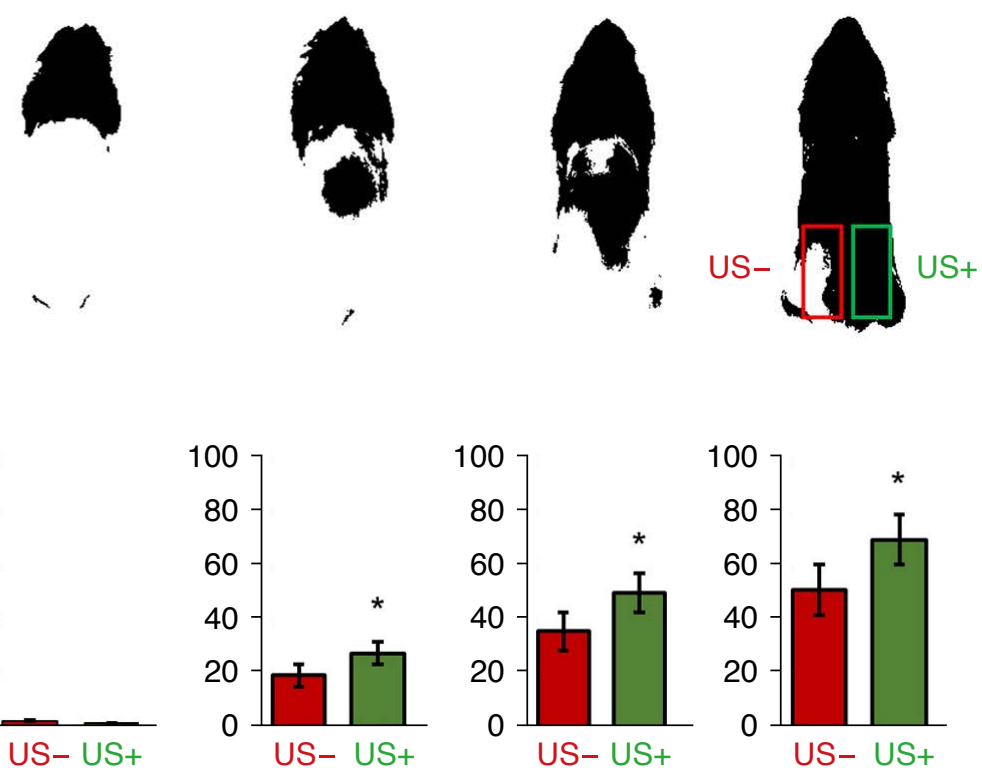

Fig. 4. Group-level time-progression of the hair growth and image analysis.

A. A composite color image of the mice $(n=11)$ indicates the degree of hair growth during 5 -week monitoring period. The photographs were taken from the same perspective distance from the mice. To create a composite image at each time point, the images were co-registered along the midline of the mice with respect to the root of the tail, and the image transparency was adjusted to $91 \%$ per mouse. The region-ofinterest used to quantify hair growth, with or without transcutaneous application of ultrasound, are marked with colored rectangles (in red, noted as 'US-', and in green noted as 'US+', respectively). B. The corresponding composite images in 8-bit grayscale are shown (pixel values $0-255)$. C. The grayscale composite images were thresholded at half of the maximum intensity to make a binary image to accentuate the area showing prevalent hair growth in the right thigh area that received sonication. D. Image-based region-of-interest analyses of the area occupying the hair growth (the ratio of binary area within the rectangular region-of-interest, being represented as the percentage hair growth) showed significant differences between the two thigh areas starting in week 3 (paired $t$ test, ${ }^{*} P<0.05$ ). Error bars indicate standard error. $L$, left; $R$, right. 
Transverse section
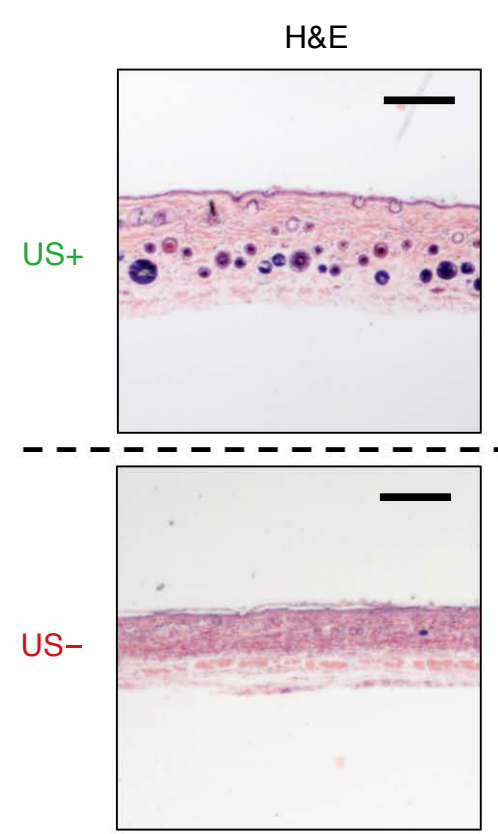
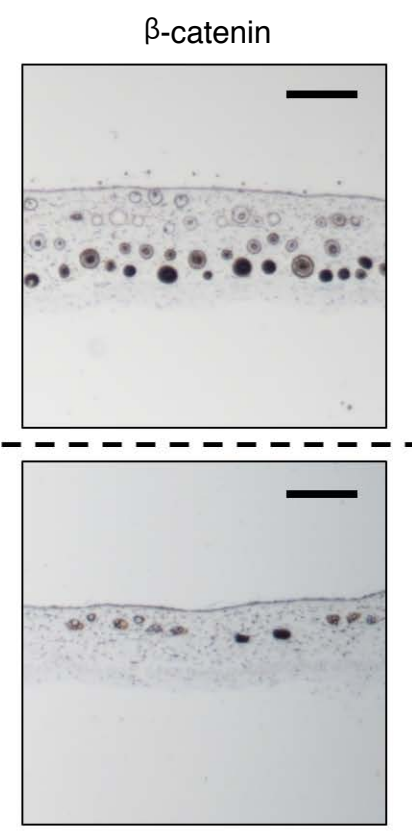

Longitudinal section

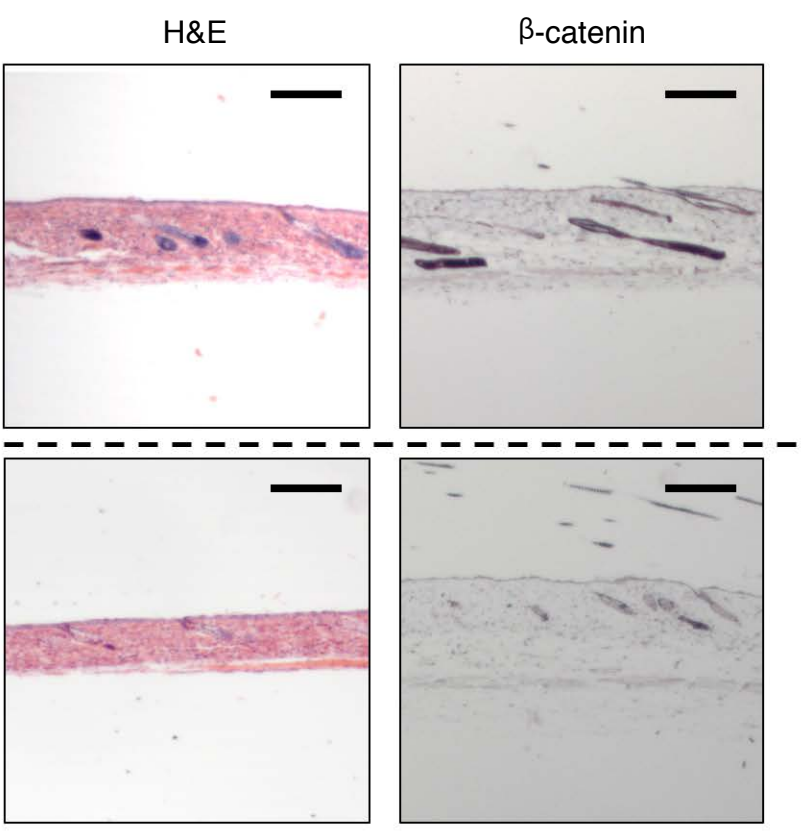

Fig. 5. Hematoxylin and eosin and $\beta$-catenin staining images $(\times 20)$ of the dorsal skin sections with (noted as 'US+') or without the transcutaneous application of ultrasound ('US-') are shown in the transverse and longitudinal sections. A higher number and larger diameter of hair follicles, along with thicker skin, were observed in the skin sections that received sonication than in the area without application of ultrasound. Scale bars $=200 \mu \mathrm{m}$.

sonicated regions.

\section{Skin Temperature Measurements and Thermal Simulation}

Fig. 7A shows a plot of skin temperature measured at the skin during sonication. There was no significant temperature change over the course of sonication (one-way repeated-measures analysis of variance: $F=0.94, P=0.432$ ). The thermal simulation (Fig. 7B, C) also revealed a negligible temperature rise in the muscle and skin $\left(\leq 0.03^{\circ} \mathrm{C}\right)$, confirming the skin temperature measurements. The results suggest that the application of low-intensity ultrasound does not elevate skin temperature.

\section{Discussion}

The present study demonstrated that the transcutaneous application of ultrasound pressure waves to the skin promoted hair growth in AA mice receiving finasteride. There was a degree of variability in the hair regrowth rate depending on the animal, and many animals failed to completely regrow their hair by the end of the monitoring period (week 5), which has been observed in previous studies using murine models of AA [29]. Nonetheless, hair began to appear on the mid-dorsal area in the back as well as on the thigh area starting on week 2, and progressively became more evident with time. The authors surmise that increasing the monitoring time window beyond a 5-week period will help show the effects of ultrasound more clearly.

Based on the histological analysis, the number and diameter of the hair follicles were greater in the sonicated area than in the unsonicated area, which agreed well with the photographic analysis. The thicker skin and higher $\mathrm{A} / \mathrm{T}$ ratio at the sonicated area suggest an increased number of anagen hair follicles, indicating enhanced hair growth.

As to the potential mechanism underlying these observations, the authors speculate that ultrasound-mediated unbinding of finasteride from the plasma albumin was the primary contributing factor. Plasma protein binding (PPB) is known to significantly influence the pharmacokinetics of drugs [30]. Many types of drugs bind to plasma proteins, such as albumin, a1-acid glycoprotein, and lipoprotein [30]. Previous studies showed that finasteride has a high affinity to plasma albumin [31], and the main binding forces between finasteride and albumin were found to be van der Waals forces via a spectrofluorimetry [32]. When finasteride binds to albumin, the large size of albumin $(66.5 \mathrm{kDa})$ means that it is not typically transported across the capillary to the interstitial space; instead, it remains in the blood, reducing the bioavailability of the drug. Increasing the systemic dose of finasteride is an option to elevate the unbound 

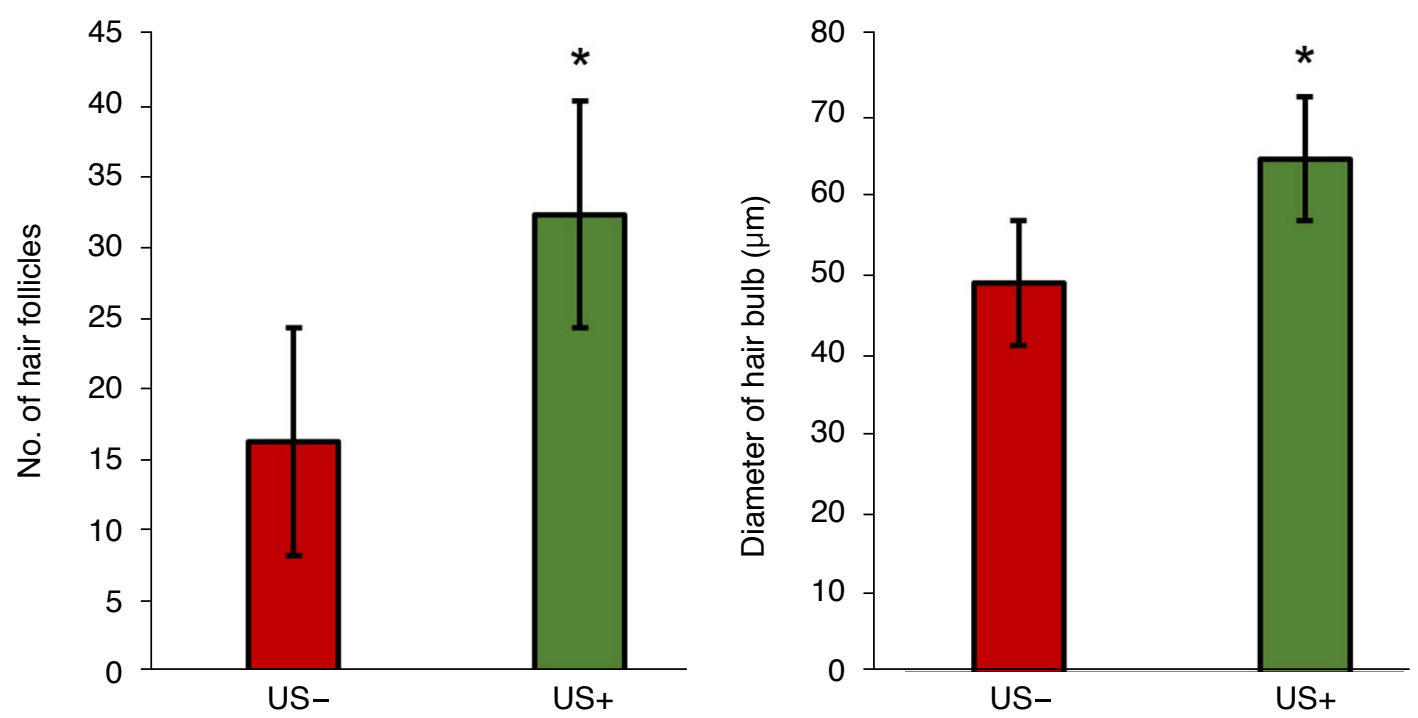

A

B
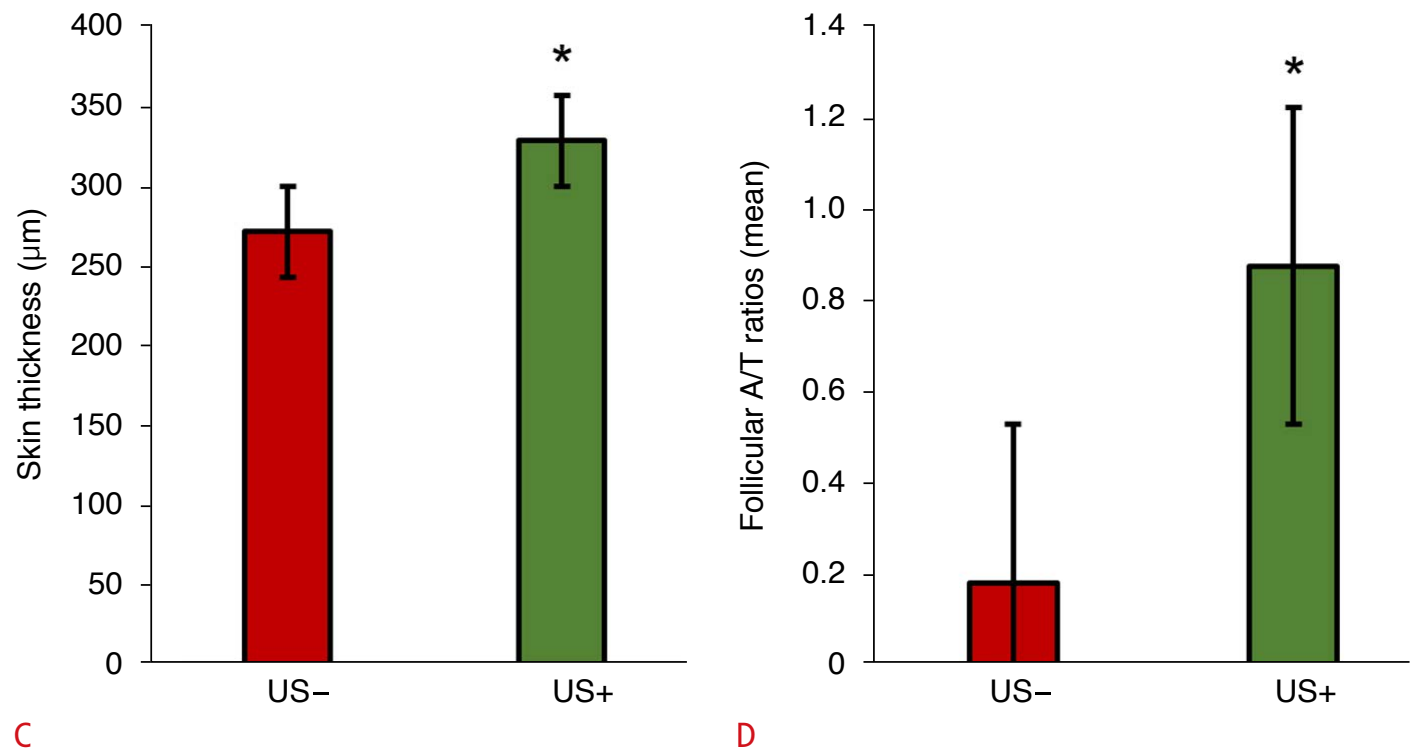

Fig. 6. Analysis of skin histology.

A. Ultrasound application increased the number of hair follicles ( $n=11$ mice) from the skin that was exposed to sonication ('US+', in the green bar) compared to the area without exposure to sonication ('US-', in the red bar) under a finasteride regimen. B. The diameter of hair follicles was greater in the sonicated skin. C. The sonication resulted in increased skin thickness. D. The anagen/telogen ratio (A/T ratio), which indicates the potential for new hair growth, was also greater in the sonicated skin. ${ }^{*} \mathrm{P}<0.05$, paired t test; error bars indicate standard error.

drug concentration to promote its action, but would increase the risk of various side effects.

As van der Waals forces are significantly weaker (on the order of piconewtons $\left[10^{-12} \mathrm{~N}\right]$ ) than ionic or covalent bonds [33], the radiation force imposed by the applied ultrasound pressure waves was strong enough to unbind finasteride from albumin. Although a direct derivation of the acoustic radiation force on the albuminfinasteride complex is difficult since its acoustic absorption coefficient is unknown, a qualitative estimation was performed based on the dimensions of albumin (roughly ellipsoid, $\sim 3 \mathrm{~nm}$ in diameter and 14 $\mathrm{nm}$ long) [34]. The size of finasteride was not considered due to its small molecular weight. The maximum surface area exposed to the incident pressure wave was approximated as $51 \times 10^{-18} \mathrm{~m}^{2}$ (estimated from half of the ellipsoidal surface). Assuming full absorption of the pressure waves $\left(549 \mathrm{kPa}\right.$, i.e., $\left.5.49 \times 10^{5} \mathrm{~N} / \mathrm{m}^{2}\right)$ at the surface, the radiation force imposed on albumin would be $2.8 \times 10^{-11} \mathrm{~N}$ 


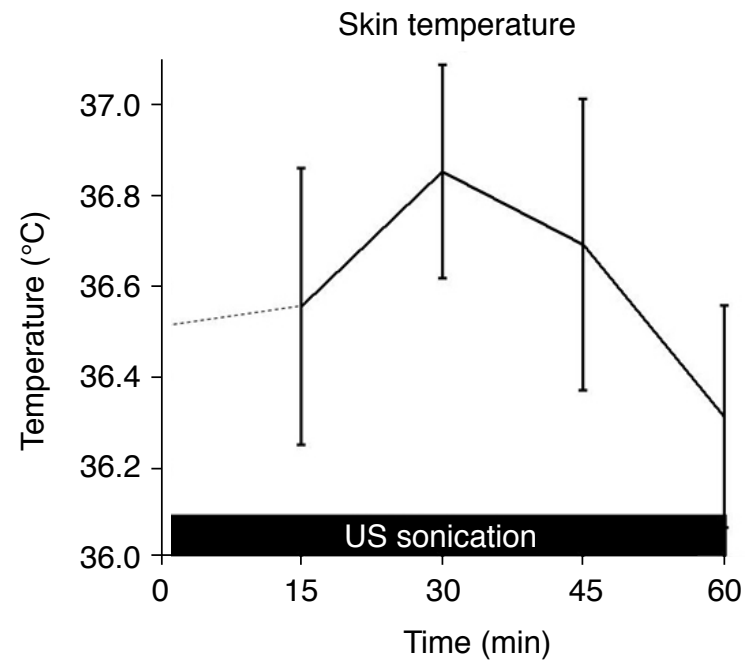

A

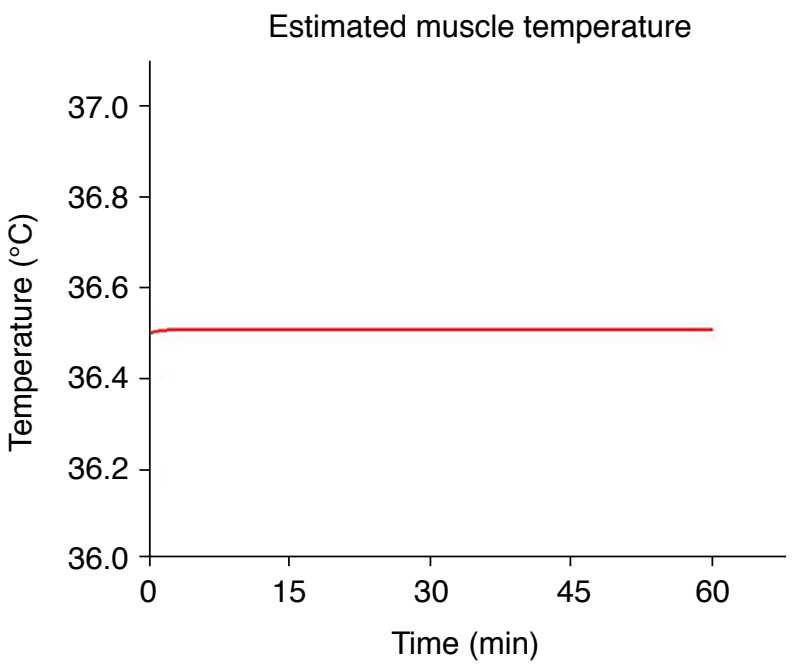

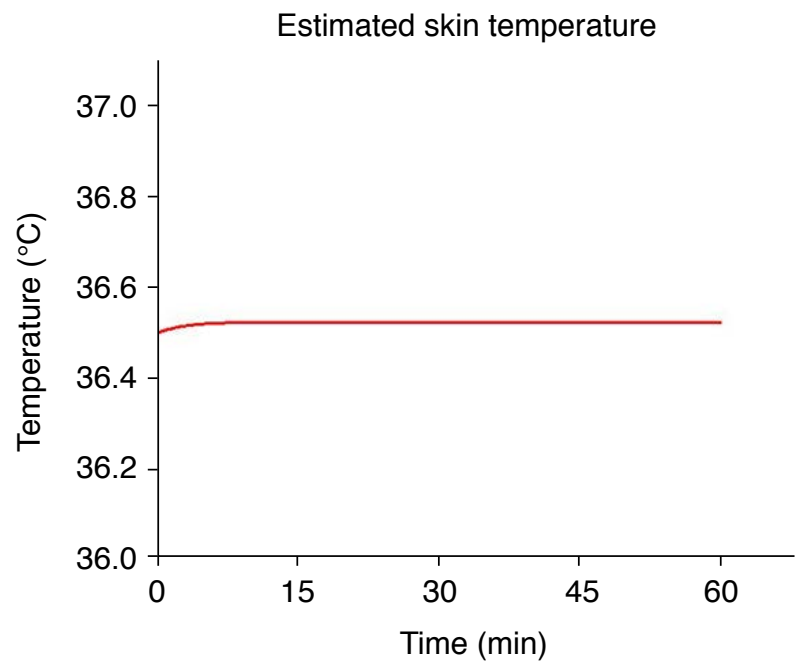

Fig. 7. Evaluation of the potential thermal effects of sonication.

A. The skin temperature measured over the course of sonication is shown (error bars: standard error). The initial skin temperature before the procedure was measured to be $36.5^{\circ} \mathrm{C}$ (dotted line) and remained unchanged (one-way repeated-measures analysis of variance, $\mathrm{F}=0.94$, $\mathrm{P}=0.432$ ). B. Numerical estimation of temperature at the skin that was exposed to a maximum in situ acoustic intensity of $2.5 \mathrm{~W} / \mathrm{cm}^{2} \mathrm{I}_{\text {SPA }}$ for 1 hour showed a negligible temperature change $\left(\leq 0.03^{\circ} \mathrm{C}\right)$. C. The estimated temperature change in the muscle was also negligible, supporting the data from skin temperature measurements and estimations.

C

(pressure $\times$ area $=5.49 \times 10^{5} \mathrm{~N} / \mathrm{m}^{2} \times 51 \times 10^{-18} \mathrm{~m}^{2}$ ), which is far greater than the binding force. Therefore, acoustic radiation force provides a plausible explanation for the observed phenomena, and the authors speculate that ultrasound sonication may temporarily and reversibly disrupt the finasteride-albumin binding, which in turn increases the available local concentration of unbound finasteride for enhanced hair growth in AA mice.

The thermal effects of sonication were excluded, as evident from the temperature measurements and thermal simulation (Fig. 7). Cavitation does not occur at the intensity and pressure used, since a pressure of $13.5 \mathrm{MPa}$ is needed to induce cavitation [35], which is about 25 times stronger than that used in the present study. Moreover, the mechanical index of 0.225 was much lower than the regulatory limit of 1.9 for most ultrasound imagers [23]. The skin, other than induced alopecia, remained normal throughout the intervention, which indicates the preliminary safety of the method.

This study has several limitations. First, the current study lacks an evaluation of sonication effects that are independent of the use of finasteride. Although the application of ultrasound (alone) without administration of finasteride would not be likely to induce any positive impact on hair growth, the inclusion of such an experimental condition would help isolate the potential effects of ultrasound pressure waves for the treatment of AA. Second, it is important to note that testosterone enanthate, which was used for inducing $\mathrm{AA}$, also strongly binds to a specific testosterone-estradiol binding globulin ( 98\%), while it weakly binds to albumin [36]; thus, ultrasound may have also enhanced the effects of testosterone enanthate, counteracting/compromising the effects of finasteride unbinding. The pair-wise comparisons of hair growth in each animal under the same experimental condition suggest that the 
unbinding of finasteride from plasma proteins by sonication might have outweighed the effects of unbinding testosterone enanthate. Nonetheless, direct in vivo assays of unbound levels of finasteride/ testosterone enanthate concentrations at the mouse skin would provide more conclusive information regarding the effects of ultrasound. Recent advances in high-resolution subcellular in vivo imaging methods have enabled more detailed analyses on how unbound drugs work at the tissue or cellular level [37], and such an analysis could be planned in future studies. In vitro equilibrium dialysis of solutions containing physiological concentrations of finasteride, albumin, and testosterone enanthate under sonication would also help to obtain quantitative data on the effects of sonication.

Another limitation of this study is the lack of a systematic assessment of the effects of varying sonication parameters (frequency and pulsing schemes) on hair growth. For example, the wavelength of ultrasound is one of the key determinants of the rate of absorption into the tissue. A higher frequency than that of $1 \mathrm{MHz}$ used in the current work would have a shorter penetration depth, depositing more energy to the skin. The pulsing scheme is another important element to maximize the radiation force to the biological tissue without heating it. Concurrently, further investigations are needed to determine the parameter set for maximal unbinding of a drug from plasma proteins to translate the technique for dermatological applications.

In summary, this study presents a novel ultrasound technique that promotes hair growth in a non-invasive manner. Ultrasound delivered to the back of the mouse skin following finasteride oral gavage treatment led to significantly higher levels of hair follicle regeneration than in unsonicated control areas. These findings position ultrasound as a useful therapeutic tool for the treatment of $A A$, through enhancement of localized delivery of finasteride without elevating the systemic drug dose (and the accompanying side effects). This approach would be especially beneficial for treating $A A$ in the presence of marginal effects on hair growth at lower dose of finasteride. The use of ultrasound to non-invasively and locally alter the PPB of drugs may impact several clinical fields. Integration of the presented technique in conjunction with other antibacterial/antifungal dermatological drugs that bind heavily to plasma proteins, for example, minocycline (for acne treatment with protein binding of $76 \%$ [38]) or itraconazole (for treatment of athlete's foot with protein binding of $99.8 \%$ [39]), may be beneficial to reduce the dose-dependent side effects, such as hepatotoxicity, commonly shared between these two drugs [40]. Specifically, in skin diseases, ultrasound may serve as a non-pharmacological treatment option, perhaps even as a complementary technique alongside existing therapeutic modalities.
ORCID: Jaeho Kim: https://orcid.org/0000-0003-3770-2359; Hyun-Chul Kim: https:// orcid.org/0000-0001-7943-3295; Kavin Kowsari: https://orcid.org/0000-0001-98059454; Kyungho Yoon: https://orcid.org/0000-0003-0193-0616; Seung-Schik Yoo: https://orcid.org/0000-0002-5150-9857

\section{Author Contributions}

Conceptualization: Yoo SS. Data acquisition: Kim J, Kim HC, Yoo SS. Data analysis or interpretation: Kim J, Kim HC, Kowsari K, Yoon K, Yoo SS. Drafting of the manuscript: Kim J, Kim HC, Kowasari K, Yoon K, Yoo SS. Approval of the final version of the manuscript: all authors.

\section{Conflict of Interest}

No potential conflict of interest relevant to this article was reported.

\section{Acknowledgments}

The study was supported by a sponsored research agreement with Deepson Bio, Ltd. and grants from the Korea Health Technology R\&D Project through the Korea Health Industry Development Institute (KHIDI), the Ministry of Health \& Welfare (grant number: HI19C1082), and Korean Neurological Association (KNA-20-NeuroFrontier Fellowship Award Grant-3).

\section{References}

1. Phillips TG, Slomiany WP, Allison R. Hair loss: common causes and treatment. Am Fam Physician 2017;96:371-378.

2. Starace M, Alessandrini A, Brandi N, Piraccini BM. Preliminary results of the use of scalp microneedling in different types of alopecia. J Cosmet Dermatol 2020;19:646-650.

3. Yao G, Jiang D, Li J, Kang L, Chen S, Long Y, et al. Self-activated electrical stimulation for effective hair regeneration via a wearable omnidirectional pulse generator. ACS Nano 2019;13:12345-12356.

4. Lanzafame RJ, Blanche RR, Bodian AB, Chiacchierini RP, FernandezObregon A, Kazmirek ER. The growth of human scalp hair mediated by visible red light laser and LED sources in males. Lasers Surg Med 2013;45:487-495.

5. Li M, Marubayashi A, Nakaya Y, Fukui K, Arase S. Minoxidil-induced hair growth is mediated by adenosine in cultured dermal papilla cells: possible involvement of sulfonylurea receptor $2 B$ as a target of minoxidil. J Invest Dermatol 2001;117:1594-1600.

6. Goren A, Naccarato T, Situm M, Kovacevic M, Lotti T, McCoy J. Mechanism of action of minoxidil in the treatment of androgenetic alopecia is likely mediated by mitochondrial adenosine triphosphate synthase-induced stem cell differentiation. J Biol Regul Homeost Agents 2017;31:1049-1053.

7. Arca E, Acikgoz G, Tastan HB, Kose O, Kurumlu Z. An open, randomized, comparative study of oral finasteride and $5 \%$ topical minoxidil in male androgenetic alopecia. Dermatology 2004;209:117-125. 
8. Saraswat A, Kumar B. Minoxidil vs finasteride in the treatment of men with androgenetic alopecia. Arch Dermatol 2003;139:12191221.

9. Inui S, Itami S. Molecular basis of androgenetic alopecia: from androgen to paracrine mediators through dermal papilla. J Dermatol Sci 2011;61:1-6.

10. Premanand A, Reena Rajkumari B. Androgen modulation of Wnt/ beta-catenin signaling in androgenetic alopecia. Arch Dermatol Res 2018:310:391-399.

11. Mysore V. Finasteride and sexual side effects. Indian Dermatol Online J 2012;3:62-65.

12. Mysore V, Shashikumar BM. Guidelines on the use of finasteride in androgenetic alopecia. Indian J Dermatol Venereol Leprol 2016;82:128-134.

13. Etzioni RD, Howlader N, Shaw PA, Ankerst DP, Penson DF, Goodman $P J$, et al. Long-term effects of finasteride on prostate specific antigen levels: results from the prostate cancer prevention trial. J Urol 2005;174:877-881.

14. Baig MS, Kolasa-Wolosiuk A, Pilutin A, Safranow K, BaranowskaBosiacka I, Kabat-Koperska J, et al. Finasteride-induced inhibition of 5alpha-reductase type 2 could lead to kidney damage-animal, experimental study. Int J Environ Res Public Health 2019;16:1726.

15. Xu L, Lee W, Rotenberg A, Bohlke M, Yoon K, Yoo SS. Localized disruption of blood albumin-phenytoin binding using transcranial focused ultrasound. Ultrasound Med Biol 2020;46:1986-1997.

16. Kim HC, Lee W, Bohlke M, Yoon K, Yoo SS. Focused ultrasound enhances the anesthetic effects of topical lidocaine in rats. BMC Anesthesiol 2021;21:158.

17. Lemke TL, Williams DA. Foye's principles of medicinal chemistry. 6 th ed. Philadelphia, PA: Lippincott Williams \& Wilkins, 2008.

18. Liao AH, Lin $\mathrm{KH}$, Chuang $\mathrm{HC}$, Tsai $\mathrm{CH}$, Lin $\mathrm{YC}$, Wang $\mathrm{CH}$, et al. Low-frequency dual-frequency ultrasound-mediated microbubble cavitation for transdermal minoxidil delivery and hair growth enhancement. Sci Rep 2020;10:4338.

19. Cho S, Shin MH, Kim YK, Seo JE, Lee YM, Park CH, et al. Effects of infrared radiation and heat on human skin aging in vivo. J Investig Dermatol Symp Proc 2009;14:15-19.

20. Klifto KM, Dellon AL, Hultman CS. Prevalence and associated predictors for patients developing chronic neuropathic pain following burns. Burns Trauma 2020;8:tkaa011.

21. Muller-Rover $S$, Handjiski B, van der Veen $C$, Eichmuller $S$, Foitzik K, McKay IA, et al. A comprehensive guide for the accurate classification of murine hair follicles in distinct hair cycle stages. J Invest Dermatol 2001;117:3-15.

22. Rastrelli G, Reisman Y, Ferri S, Prontera O, Sforza A, Maggi M, et al. Testosterone replacement therapy. In: Gunasekaran K, Khan S, eds. Sexual medicine: principles and practice. Singapore: Springer Singapore, 2019;79-93.

23. Lee W, Weisholtz DS, Strangman GE, Yoo SS. Safety review and perspectives of transcranial focused ultrasound brain stimulation. Brain Neurorehabil 2021;14:e4.

24. Huelsken J, Vogel R, Erdmann B, Cotsarelis G, Birchmeier W. Beta-catenin controls hair follicle morphogenesis and stem cell differentiation in the skin. Cell 2001;105:533-545.

25. Klemp P, Peters K, Hansted B. Subcutaneous blood flow in early male pattern baldness. J Invest Dermatol 1989;92:725-726.

26. Okajima J, Maruyama S, Takeda H, Komiya A. Dimensionless solutions and general characteristics of bioheat transfer during thermal therapy. J Therm Biol 2009;34:377-384.

27. Soneson JE. A user-friendly software package for HIFU simulation. AIP Conf Proc 2009;1113:165-169.

28. Dallapiazza RF, Timbie KF, Holmberg S, Gatesman J, Lopes MB, Price $\mathrm{RJ}$, et al. Noninvasive neuromodulation and thalamic mapping with low-intensity focused ultrasound. J Neurosurg 2018;128:875-884.

29. Kim JH, Na J, Bak DH, Lee BC, Lee E, Choi MJ, et al. Development of finasteride polymer microspheres for systemic application in androgenic alopecia. Int J Mol Med 2019;43:2409-2419.

30. Bohnert T, Gan LS. Plasma protein binding: from discovery to development. J Pharm Sci 2013;102:2953-2994.

31. Sudduth SL, Koronkowski MJ. Finasteride: the first 5 alphareductase inhibitor. Pharmacotherapy 1993;13:309-325.

32. Abdelhameed AS, Alanazi AM, Kadi AA. Spectrofluorimetric study of finasteride and bovine serum albumin interaction and its application for quantitative determination of finasteride in tablet dosage form. Anal Methods 2015;7:5096-5102.

33. Shi JH, Pan DQ, Jiang M, Liu TT, Wang Q. Binding interaction of ramipril with bovine serum albumin (BSA): insights from multispectroscopy and molecular docking methods. J Photochem Photobiol B 2016;164:103-111.

34. Kiselev MA, Gryzunov lu A, Dobretsov GE, Komarova MN. Size of a human serum albumin molecule in solution. Biofizika 2001;46:423427.

35. Lin KW, Kim Y, Maxwell AD, Wang TY, Hall TL, Xu Z, et al. Histotripsy beyond the intrinsic cavitation threshold using very short ultrasound pulses: microtripsy. IEEE Trans Ultrason Ferroelectr Freq Control 2014;61:251-265.

36. Goldman AL, Bhasin S, Wu FC, Krishna M, Matsumoto AM, Jasuja R. A Reappraisal of testosterone's binding in circulation: physiological and clinical implications. Endocr Rev 2017;38:302-324.

37. Thurber GM, Yang KS, Reiner T, Kohler RH, Sorger P, Mitchison T, et al. Single-cell and subcellular pharmacokinetic imaging allows insight into drug action in vivo. Nat Commun 2013;4:1504.

38. Macdonald H, Kelly RG, Allen ES, Noble JF, Kanegis LA. Pharmacokinetic studies on minocycline in man. Clin Pharmacol Ther 1973;14:852-861.

39. Schafer-Korting M, Korting HC, Amann F, Peuser R, Lukacs A. Influence of albumin on itraconazole and ketoconazole antifungal activity: results of a dynamic in vitro study. Antimicrob Agents 
Chemother 1991;35:2053-2056.

40. Somchit N, Norshahida AR, Hasiah AH, Zuraini A, Sulaiman MR, Noordin MM. Hepatotoxicity induced by antifungal drugs itraconazole and fluconazole in rats: a comparative in vivo study. Hum Exp Toxicol 2004;23:519-525. 